\title{
APPLICATIONS OF ZIGBEE WIRELESS TECHNOLOGY TO MEASUREMENT SYSTEM IN GRAIN STORAGE
}

\author{
Huiling Zhou ${ }^{1, *}$, Xuechuan Chen ${ }^{1}$, Xiangdong Liu ${ }^{2}$, Jun Yang ${ }^{1}$ \\ ${ }^{1}$ Automation School, Beijing University of Posts and Telecommunication, Beijing, China, \\ 100876 \\ ${ }^{2}$ College of Engineering, China Agricultural University, Beijing, China, 100083 \\ * Corresponding author, Address: P. O. Box 107, Automation School, Beijing University \\ of Posts and Telecommunication, Beijing, 100876, P.R.China, Tel: +86-10-62282927, \\ Fax:+86-10-62282629, Email: huiling@bupt.edu.cn
}

\begin{abstract}
Wireless sensor network (WSN) has attracted more and more attentions in the computer science and communication research community. It has appeared a great potential in numerous industrial applications. In this paper a wireless sensor network based on ZigBee protocol for the measurement system in grain storage was developed and tested as an alternative to the cable system, which showed better efficiencies of the cost and time in the installation and maintenance than a cable system. Based on above study a new ZigBee sensornode was built to measure the grain temperature and the temperature, moisture and pressure drop of the air through a bulk grain system. The wireless data transmission and the power consumption were tested. This paper proposed a practical solution for the ZigBee protocol.
\end{abstract}

Keywords: wireless sensor network, ZigBee, grain storage, date transmission, power consume

\section{INTRODUCTION}

The scientific researches have already shown that the grain quality in the barn was strongly related to the grain storage condition, the environment and the storage time (Li Tiepan et al., 2004). An effective monitoring of temperature and humidity is fundamental for ensuring the grain quality

Please use the following format when citing this chapter:

Zhou, H., Chen, X., Liu, X. and Yang, J., 2009, in IFIP International Federation for Information Processing, Volume 295, Computer and Computing Technologies in Agriculture II, Volume 3, eds. D. Li, Z. Chunjiang, (Boston: Springer), pp. 2021-2029. 
during the storage. According to the real time information of grain temperature and humidity, the aeration, as well as optimal aeration time, are necessary to inhibit from microbial growth, sprouting and germination and insect population growth.

Wireless Sensor Network (WSN) is a system comprised of radio frequency (RF) transceivers, sensors, microcontroller and power source. This system with self-organizing, self-configuring, self-diagnosing and selfhealing capability has been developed to solve problems or to enable application that traditional technologies could not address. WSN originates from military and environmental monitoring, moves towards machine to machine communication and is eventually applied to all aspects of our life (Wang, N. et al., 2006). Compared with the fieldbus system, WSN for monitoring has the following advantages: simple structure, less problems than connecting wires; feasibility and low cost of installation; and reliable and stable communication (Maxwell, D. et al., 2002).At the present, ZigBee within ISM band of $2.4 \mathrm{GHz}$ based on provided license-free operations, huge spectrum allocation and worldwide compatibility, has been specifically developed to address the demands of wireless sensor networks, including the need to handle a large number of nodes (more than 65,000). ZigBee built upon the physical (PHY) and medium access control (MAC) layers defined by the IEEE 802.15.4 standard (IEEE, 2003) enables the creation of a higher network flexibility, such as star, cluster tree or mesh network, and is very suitable for industrial, agricultural, vehicular, residential and medical environments. The intent of IEEE 802.15.4 is to provide an ultra low power consumption and very short wake-up time capabilities at very low cost to devices operating in a Low Rate $(250 \mathrm{kbps})$ Wireless Personal Area Network (LR-WPAN). The IEEE 802.15.4 assumes that the data transmitted are short and that transmissions occur at a low-duty cycle (active/sleep times ratio), reducing the overall power needs and enabling the application of batterypowered embedded systems (Raul Morais et al., 2008).

Wireless sensor technology based on ZigBee protocol is still at its early development stage. Applications in grain storage are very limited. This paper is to give a kind of ZigBee node (Tech1) for monitoring the grain temperature, the temperature-humidity and pressure of the air through the bulk grains during storage. The node's hardware architecture and the experiments to get the data of the temperature, temperature-humidity, transmission distance and the battery life are major issues studied in this work. 


\section{HARDWARE OVERVIEW OF ZIGBEE NODE}

ZigBee node Tech1 is integrated by a microcontroller board (ZigBee board) and a sensor board with DC-DC transducer (3V-3V, 3V-5V). Node Tech 1 architecture prototype is shown in Fig.1.

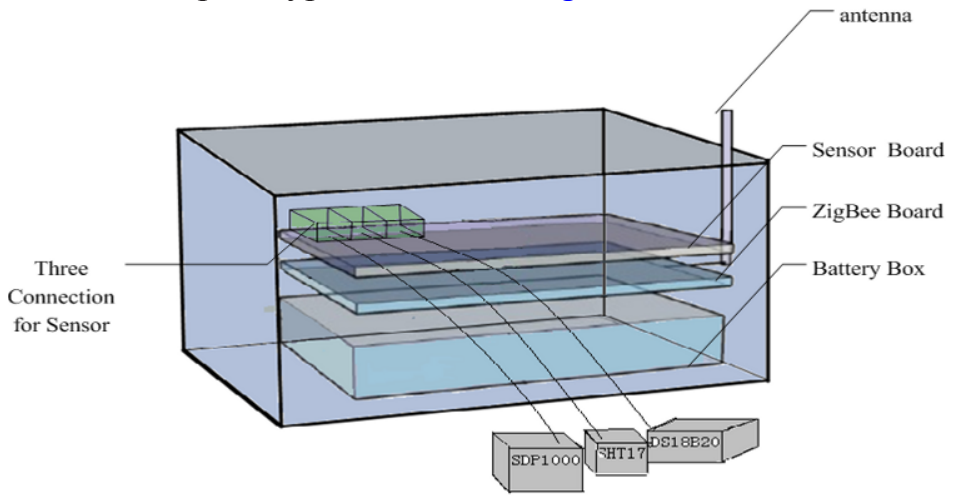

Fig. 1. ZigBee node Tech1 architecture prototype

The ZigBee board hosts a TI CC2430 that is a true SoC solution tailored for IEEE802.15.4 and ZigBee applications. An enhanced 8051 microcontroller core, a $14 \mathrm{bitA} / \mathrm{D}$, a $128 \mathrm{MB}$ flash and a $8 \mathrm{kB}$ RAM are embedded in the CC2430. The CC2430 also combines CC2420 RF transceiver, and combined with the industry leading ZigBee protocol stack (Datasheet for SmartRF ${ }^{\circledR}$ CC2430, 2005). It is specifically suited for the monitoring system of grain storage for which ultra low power consumption is required.

Sensor board consists of the sensors and DC-DC model. And three types of precise digital sensors are used: a maxim DB18B20 for monitoring the grain temperature, a Sensirion SHT71 for measuring the temperature and moisture of the air through the bulk grains, and a Sensirion SDP1000 for collecting the pressure information through the bulk grains during the aeration. These wireless sensors have signal conditioning and processing units and transmit signals in the digital form, therefore the noise pick-up becomes a less significant problem. The sensor block diagrams are given in Fig.2 (Datasheet for DS18B20, Datasheet for SHTxx(v2.04) and SDP1000). 


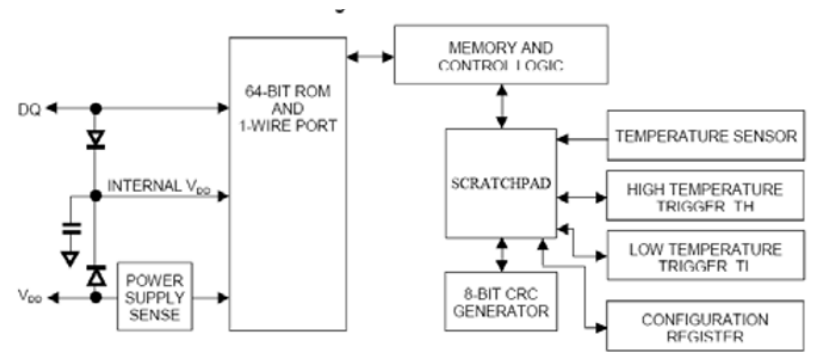

(a) DB18B20 block diagram

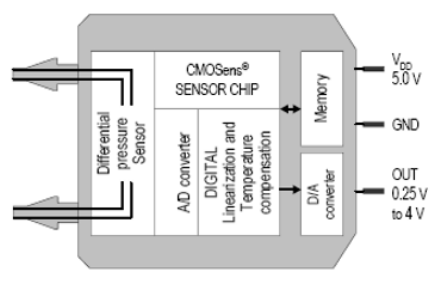

(b) SDP1000 block diagram

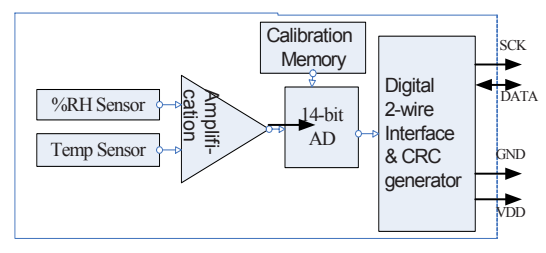

(c) SHT71 bolck diagram

Fig. 2. Sensor block diagrams

The node power comes from two $1.5 \mathrm{~V}$ AAA batteries. Because the ZigBee board and sensors need different voltages to provide power, the compact and high efficient DC-DC converter (Maxim Max1677) was designed. The input voltage range is $+0.7-+5.5 \mathrm{~V}$, the output voltage is $3 \mathrm{~V}$ to ZigBee board and sensor SHT71, 5V to sensors DB18B20 and SDP1000. The photograph of Tech1 node is shown in Fig.3.

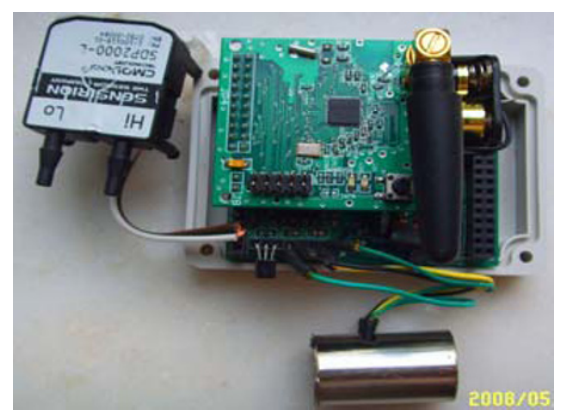

Fig.3. Sensor block diagrams

\section{EXPERIMENT OF LABORATORY AND RESULTS}

In order to test the node work reliability and the transmission distance through grain, a ZigBee star network with 4 Tech1 nodes is constructed in the laboratory (see Fig.4): one is the full function device(FFD) as a 
coordinator, three are reduced function devices (RFD) as end devices. This categorization makes power saving possible, because RFD devices need to be connected to the network only to check network messages and transmit sensor data to coordinator. A monitoring system runs in PC, which communicates with the coordinator by RS232 interface, in order to display the information of the temperature, humidity, pressure, and RSSI data from each node timely, as well as battery voltage. As test material, the steel grain bin is filled with millet.

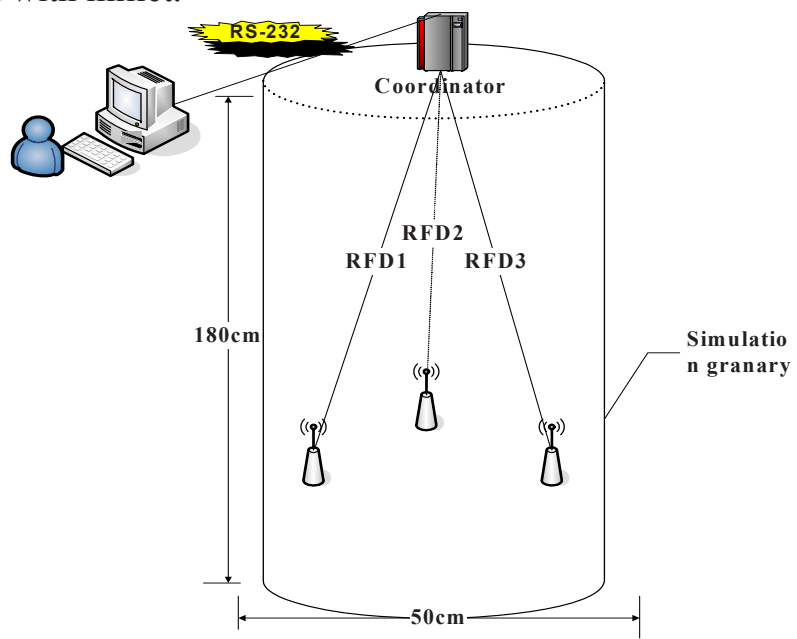

Fig.4: End devises and coordinator placement within grain

\subsection{Distance estimation}

In order to estimate the transmission distance of Tech1 within the grain, Received Signal Strength Indication (RSSI) technology is used. RSSI measures the attenuation in radio signal strength between the coordinator and the end devise. Although RSSI technology for distance estimation is not very inaccurate(J. Hightower et al., 2000), this data is mainly used to get approximate maximum grain depth that the node can communicate and, as well as to estimate the total nodes of the real ZigBee wireless monitoring network within grain in the future.

Distance estimation based on RSSI needn't use any extra hardware support. CC2430 has a built-in RSSI giving a digital value that can be read from the 8 bit, signed 2's complement RSSIL.RSSI_VAL register bits. The RSSI value is always averaged over 8 symbol periods $(128 \mu \mathrm{s})$, in accordance with IEEE Standards (IEEE std. 802.15.4, 2003). The RSSI register value RSSI.RSSI_VAL can be referred to the power P at the RF pins by using the following equation 


$$
P=R S S I \_V A L+R S S I \_O F F S E T[d B M]
$$

Where: RSSI_OFFSET is approximately -45 . E.g. if reading a value of 20 from the RSSI register, the RF input power is approximately $-65 \mathrm{dBm}$. A typical plot of the RSSI_VAL reading as function of input power is shown in Fig.5. It can be seen from the figure that the RSSI reading from CC2430 is very linear and has a dynamic range of about $100 \mathrm{~dB}$ (Datasheet for SmartRF ${ }^{\circledR}$ CC2430, 2005).

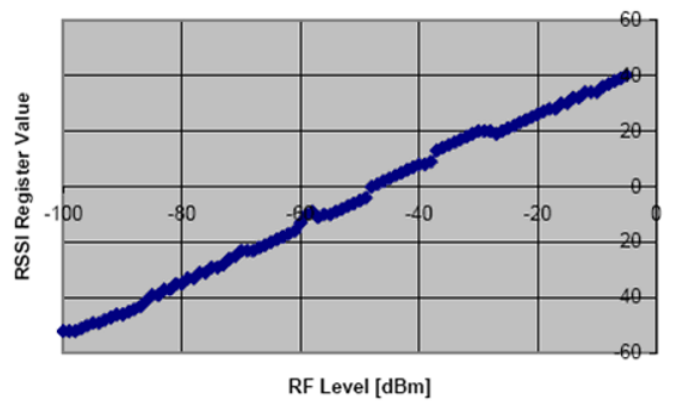

Fig.5. Typical RSSI value vs. P

In the tests, three nodes and the coordinator were first placed above the grain, and then the nodes were pushed slowly into grain, the depth and RSSI_VAL values were recorded. The average signal attenuation is shown in Fig.6. These data indicate that the node communication distance is approaching $115 \mathrm{~cm}$. Beyond this range the power of the radio signal falls off so rapidly and can't connect with network. But this result doesn't accord well with the equation given by Nelson(Nelson, S.O., 1996), with the steel grain bin the signals' high reflectivity and the relation with the direction of the antenna maybe are the reasons.
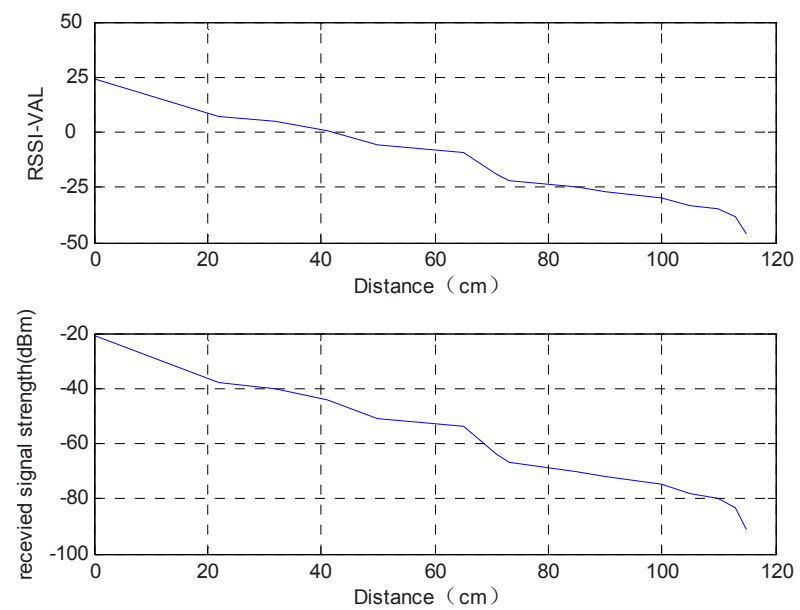

Fig.6. Signal attenuation for distance ( ambient air temperature $22.9^{\circ} \mathrm{C}, \% \mathrm{RH} 81.5 \%$ ) 


\subsection{Temperature, temperature-humidity, pressure data collection and transmission}

In order to test the node's reliability many experiments were made in the laboratory. The plots in Fig.7 are a part of data in one experiment. These data are shown that Tech1 can reliable work within grain. These results are very useful for the next research which will focus on the control of grain storage. As the plots shown that during the aeration the humidity changed very rapidly, the temperature decreased at first, and then increased gradually.
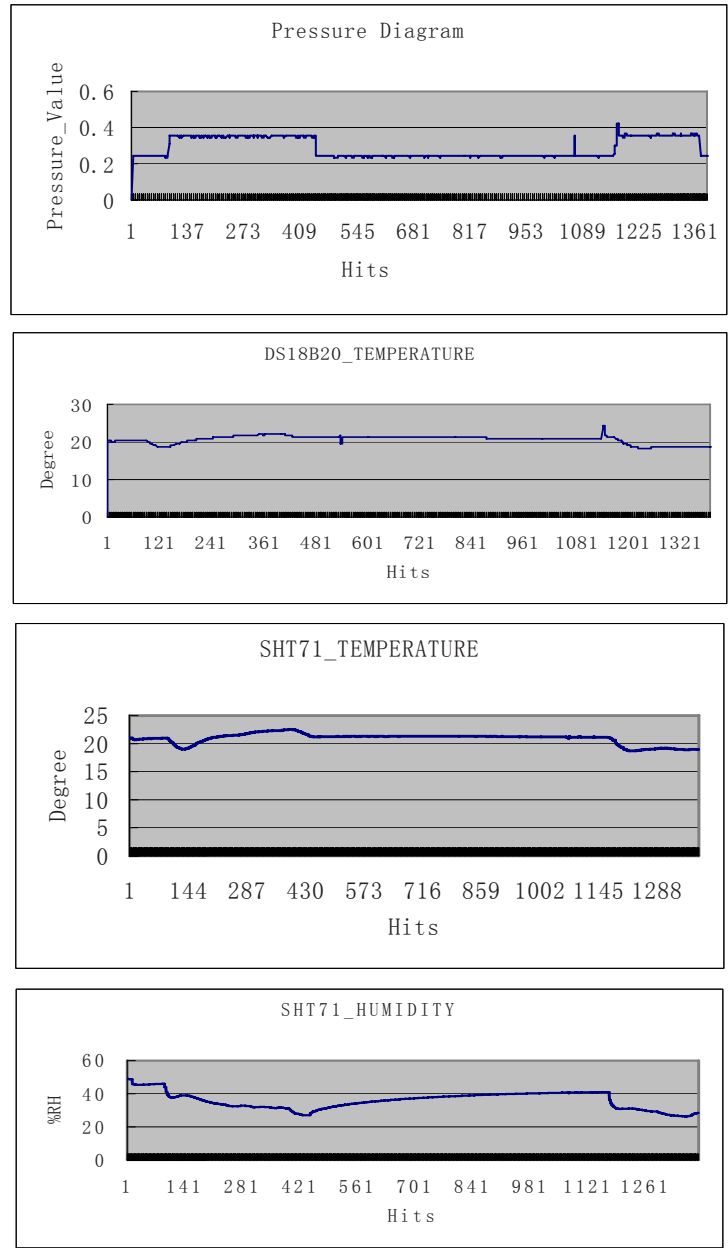

Fig.7. Temperature, temp-humidity and pressure data from Tech1(Test: sending data pre 2 second, 15:23:21-16:46:09,2008/5/10,Beijing) 


\subsection{Node lifetime estimation}

The node lifetime decides whether a grain storage monitoring network based ZigBee protocol can give an application-level solution. In order to estimate Tech1 lifetime, our test strategy is:

- One end devise with 2 new 1.5V AAA alkalescent batteries

- Battery capacity about $2200 \mathrm{mAh}$ (Mainwaring A et al., 2002)

- CC2430 MCU still active and TX mode, 0dBm

- sensors data transmission Per 2 second

In theory, the current consumption of CC2430 MCU active and TX mode is about $24.7 \mathrm{~mA}, \mathrm{ADC}$ current consumption is $0.9 \mathrm{~mA}$, Timer is $19 \mathrm{uA}$ (Datasheet for SmartRF ${ }^{\circledR}$ CC2430, 2005), DB18B20 work current is $1 \mathrm{~mA}$, SHT71 work current is $550 \mathrm{uA}$ (voltage source $5 \mathrm{~V}, \mathrm{Temp} .25^{\circ} \mathrm{C}$ ) and SDP1000 work current is 5mA (Datasheet for SHTxx(v2.04) and SDP1000). Because the work time durations of these digital sensors are all less than $1 \mathrm{~ms}$, and then transfer into sleep state that power consumption is very low. The time of data sending one time of the end devise is also less than $1 \mathrm{~ms}$, the node total power consumption per one second is more lower than $31.169 \mathrm{~mA}$. As SHT71 work voltage range is $5 \mathrm{~V}-2.4 \mathrm{~V}$, the total runs needed for the end devise sending data are at least

$$
\begin{aligned}
& 440 \mathrm{mAh} / 31.169 \mathrm{mAs}=440 * 3600 / 31.62 \\
& \approx 50818 \text { (times) }
\end{aligned}
$$

In according to this work mode, the result of our node lifetime estimation experiment is: 56206 times. The test result proved the theory calculation validity.

The end devises in grain operate normally at really low duty cycles. If the network nodes wake up, perform their tasks and then revert back to sleep state, this would be very beneficial in terms of extending node lifetime.

\section{FUTURE WORK}

The ZigBee Protocol is the only international standard wireless sensor network protocol in existence, and is suitable for the special needs of lowpower, low-cost, low maintenance monitoring and control systems. In this paper the data collection and transmission were based on a simple ZigBee star network. If the sensor network is mesh network or cluster tree network topologies, routers will need to be capable of running in lower power sleep modes, waking up in time to perform any of their assigned tasks and then going back to sleep mode. Thus how to create a routing optimization mechanism to increase the network lifetime will be the future work. Another challenge work is to find the relationship between the grain temperature and 
the temperature humidity of the air through the bulk grains during the aeration.

\section{REFERENCES}

Datasheet for DS18B20. http://www.maxim-ic.com.

Datasheet for SHTxx(v2.04) and SDP1000, 2004. http://www.sensirion.com.

Datasheet for SmartRF ${ }^{\circledR}$ CC2430, 2005. http://www.maxim-ic.com.

IEEE std. 802.15.4, 2003: Wireless Medium Access Control (MAC) and Physical Layer(PHY) specifications for Low Rate Wireless Personal Area Networks (LR-WPANs).

J. Hightower, R. Want, and G. Borriello. "SpotON: An Indoor 3D Location Sensing Technology Based on RF Signal Strength" Technical Report, UW CSE 00-02-02, University of Washington, Seattle, WA, February 2000.

Li Tiepan, Song Youlin. Distribution function of temperature in grain storehouse and its application, Journal of Zhengzhou Institute of Technology, 2004, 25(3):77-79(in Chinese).

Mainwaring A, Polastre J, Szewczyk R, Culler D, Anderson J. Wireless sensor networks for habitat monitoring, In: Proc of ACM WSNA'02,2002.

Maxwell, D., Williamson, R., 2002. Wireless temperature monitoring in remote systems, sensors, Sensorsmag, October, 2002.

Nelson, S.O., 1996. Review and assessment of radio-frequency and microwave energy for stored-grain insect control. Transactions of the ASAE. 39(4): 1475-1484.

Raul Morais, Miguel A. Fernandes. Computers and electronics in agriculture $62\left(\begin{array}{llll}2 & 0 & 0 & 8\end{array}\right)$ 94-106, A ZigBee multi-powered wireless acquisition device for remote sensing applications in precision viticulture.

Wang, N., Zhang, N., Wang, M., 2006. Wireless sensors in agriculture and food industry recent development and future perspective. Computer and Electronics in Agriculture 50,114. 